\title{
Harita Projeksiyonları ve Nümerik Analiz
}

\author{
İbrahim Öztuğ BILLDIRICíi* \\ Konya Teknik Üniversitesi, Mühendislik ve Doğa Bilimleri Fakültesi \\ iobildirici@ktun.edu.tr
}

$\ddot{\mathrm{O} z}$

Harita projeksiyonları problemlerinin çözümünde çeşitli nümerik analiz yöntemlerine gerek duyulur. Doğrusal olmayan denklemlerin iteratif çözümü yaygın kullanılır. Kaynaklarda çok fazla ele alınmamasına rağmen nümerik türevin pratik olarak kullanımı mümkündür. Nümerik türev deformasyon araştırmasında, doğrusal olmayan denklem çözümleri ise ara değişken kullanılan gerçek anlamlı olmayan projeksiyonlarda ve ters projeksiyon uygulamalarında kullanılır. Bu çalışmada yöntemlerin uygulamaları örnekler üzerinde tartışılmış, parametre önerileri yapılmıştır.

Anahtar Kelimeler: Harita projeksiyonları, projeksiyon deformasyonları, nümerik analiz

\section{Map Projections and Numerical Analysis}

\begin{abstract}
There are certain numerical analysis methods that have been used in solving map projection problems. In this study methods such as numerical derivation and solving nonlinear equations are introduced, and their usage possibilities are discussed. Additionally, parameter suggestions, and special numerical approaches are given.
\end{abstract}

Keywords: Map projections, projection distortions, numerical analysis

\section{Giriş}

$\mathrm{Bu}$ makalede harita projeksiyonlarında uygulanabilecek nümerik analiz konuları tartışlacaktır.

İk olarak nümerik türev konusu ele alınacak, nümerik türev için harita projeksiyonu uygulamalarında kullanılabilecek parametrenin değeri analiz edilecektir. Daha sonra kısmi türevlerin sıklıkla kullanıldığ diferansiyel anlamda deformasyon analizi, projeksiyon yüzeyinin eğik olmasında kısmi türevlerdeki değişiklikler incelenip daha sonra nümerik ters projeksiyon uygulamaları ele alınacaktır.

\section{Harita Projeksiyonu}

Harita projeksiyonları orijinal yüzeydeki (küre ya da elipsoit) bir noktayı harita düzleminde bir noktaya izdüşüren bir fonksiyon çifti ile tanımlanır. Orijinal yüzeyden düzleme izdüşüm düz dönüşüm (forward transformation) olarak da ifade edilir. Burada kapalı bir yüzeyin düzleme dönüşümü söz konusudur.

$$
x=x(\varphi, \lambda), \quad y=y(\varphi, \lambda)
$$

$\mathrm{Bu}$ dönüşümün tersine de (düzlemden orijinal yüzeye) ihtiyaç duyulur. $\mathrm{Bu}$, ters dönüşüm (inverse transformation) olarak da adlandırılır.

$$
\varphi=\varphi(x, y), \quad \lambda=\lambda(x, y)
$$

*Sorumlu yazar 


\section{Nümerik Türev}

Bir fonksiyonun türevinin sayısal değeri gerekli olduğunda türev almaksızın nümerik olarak yaklaşık değeri bulunabilir.

$$
f^{\prime}(x)=\frac{f(x+t)-f(x)}{t}
$$

Ya da

$$
f^{\prime}(x)=\frac{f(x+t)-f(x-t)}{2 t}
$$

Burada $t$ yeterince küçük seçilecek bir sayısal değerdir. Türevin geometrik anlamı fonksiyona teğet doğrunun eğimi olduğuna göre nümerik türevde teğet doğruya kesen bir doğru ile yaklaşılmaya çalışılmaktadır. Seçilen $t$ değeri sıfıra yaklaştıkça (3) eşitliği belirsizleşir. Sifırdan uzaklaştıkça kesen doğru teğet doğrudan uzaklaşır (Şekil 1). Kısmi türevler söz konusu ise;

$$
f=f(x, y)
$$

olmak üzere,

$$
\begin{aligned}
& \frac{\partial f}{\partial x}=\frac{f(x+t, y)-f(x-t, y)}{2 t} \\
& \frac{\partial f}{\partial y}=\frac{f(x, y+t)-f(x, y+t)}{2 t}
\end{aligned}
$$

şeklinde elde edilir. Harita projeksiyonları (1) eşitlikleri ile biliniyorken kısmi türevler sayısal olarak elde edilebilir. Bu şekilde kimi zaman oldukça karmaşık olan fonksiyonların türevleri alınmaksızın türevlerin sayısal değerleri elde edilebilir.

$$
\begin{aligned}
& \frac{\partial x}{\partial \varphi}=\frac{x(\varphi+t, \lambda)-x(\varphi-t, \lambda)}{2 t} \\
& \frac{\partial x}{\partial \lambda}=\frac{x(\varphi, \lambda+t)-x(\varphi, \lambda-t)}{2 t} \\
& \frac{\partial y}{\partial \varphi}=\frac{y(\varphi+t, \lambda)-y(\varphi-t, \lambda)}{2 t} \\
& \frac{\partial y}{\partial \lambda}=\frac{y(\varphi, \lambda+t)-y(\varphi, \lambda-t)}{2 t}
\end{aligned}
$$

\section{1 İdeleme: Trigonometrik Fonksiyonlar}

Türevi hesaplanacak fonksiyonlarda trigonometrik ifadeler geçiyorsa burada $t$ parametresinin birimi radyan olmalıdır. Örneğin $f(x)=\sin x$ ise ve trigonometrik fonksiyonlarda derece kullanılacak ise,

$$
f^{\prime}(x)=\frac{\sin \left(x+t \frac{180^{\circ}}{\pi}\right)-\sin \left(x-t \frac{180^{\circ}}{\pi}\right)}{2 t}
$$

şeklinde hesap yapılmalıdır. Örneğin $t=10^{-8}, x=30^{\circ}$ alınirsa $f^{\prime}(x)=0.866025407$ bulunur. Türev alıp değerler yerine konulursa $f^{\prime}(x)=\cos x=0.866025404$ elde edilir. $\mathrm{Bu}$ örnekte nümerik türev olması gereken değerden $10^{-9}$ kadar büyük olup, olması gereken değere çok yakındır.

En uygun $t$ değeri için belli bir aralıkta nümerik türev türev farklarına bakılabilir. $\mathrm{Bu}$ amaçla $-85^{\circ}, 85^{\circ}$ aralığında sin, cos, tan fonksiyonlarında nümerik türevin türevden farkı değişik $t$ değerleri için belirlenebilir. Tablo 1, 2, 3'de $t$ değeri küçüldükçe ilgili açı aralığında $1^{\circ}$ aralıklarla hesaplanan farkların mutlak değer olarak ortalaması ve en büyük fark görülmektedir.

Tablo 1, 2 ve 3 incelendiğinde en uygun değerin $10^{-5}, 10^{-6}$ aralı̆̆ında olduğu görülmektedir. Burada $t$ değeri küçüldükçe daha uygun bir nümerik türev değerine ulaşılmadığ 1 da görülmektedir.

Tablo 1: Sinüs fonksiyonu için nümerik türev analizi

\begin{tabular}{l|l|l}
\hline$t$ & Ortalama fark & Maks. fark \\
\hline $1.00 \mathrm{e}-02$ & $1.12 \mathrm{e}-05$ & $1.67 \mathrm{e}-05$ \\
$1.00 \mathrm{e}-03$ & $1.12 \mathrm{e}-07$ & $1.67 \mathrm{e}-07$ \\
$1.00 \mathrm{e}-04$ & $1.12 \mathrm{e}-09$ & $1.67 \mathrm{e}-09$ \\
$1.00 \mathrm{e}-05$ & $1.14 \mathrm{e}-11$ & $2.19 \mathrm{e}-11$ \\
$1.00 \mathrm{e}-06$ & $2.37 \mathrm{e}-11$ & $8.34 \mathrm{e}-11$ \\
$1.00 \mathrm{e}-07$ & $2.18 \mathrm{e}-10$ & $9.22 \mathrm{e}-10$ \\
$1.00 \mathrm{e}-08$ & $2.29 \mathrm{e}-09$ & $8.72 \mathrm{e}-09$ \\
$1.00 \mathrm{e}-09$ & $2.27 \mathrm{e}-08$ & $8.31 \mathrm{e}-08$ \\
$1.00 \mathrm{e}-10$ & $1.50 \mathrm{e}-07$ & $5.27 \mathrm{e}-07$ \\
$1.00 \mathrm{e}-11$ & $1.56 \mathrm{e}-06$ & $5.40 \mathrm{e}-06$ \\
$1.00 \mathrm{e}-12$ & $2.28 \mathrm{e}-05$ & $8.64 \mathrm{e}-05$ \\
$1.00 \mathrm{e}-13$ & $2.29 \mathrm{e}-04$ & $7.86 \mathrm{e}-04$ \\
$1.00 \mathrm{e}-14$ & $1.62 \mathrm{e}-03$ & $5.97 \mathrm{e}-03$ \\
\hline
\end{tabular}



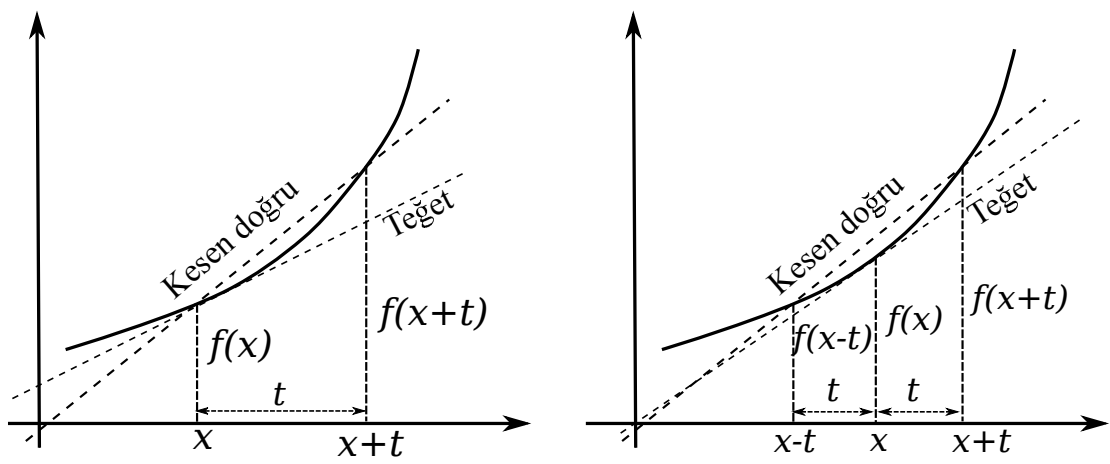

Şekil 1: Nümerik türev

Tablo 2: Kosinüs fonksiyonu için nümerik türev analizi

\begin{tabular}{l|l|l}
\hline$t$ & Ortalama fark & Maks. fark \\
\hline $1.00 \mathrm{e}-02$ & $1.02 \mathrm{e}-05$ & $1.66 \mathrm{e}-05$ \\
$1.00 \mathrm{e}-03$ & $1.02 \mathrm{e}-07$ & $1.66 \mathrm{e}-07$ \\
$1.00 \mathrm{e}-04$ & $1.02 \mathrm{e}-09$ & $1.66 \mathrm{e}-09$ \\
$1.00 \mathrm{e}-05$ & $9.44 \mathrm{e}-12$ & $2.16 \mathrm{e}-11$ \\
$1.00 \mathrm{e}-06$ & $4.12 \mathrm{e}-11$ & $1.03 \mathrm{e}-10$ \\
$1.00 \mathrm{e}-07$ & $3.66 \mathrm{e}-10$ & $9.43 \mathrm{e}-10$ \\
$1.00 \mathrm{e}-08$ & $3.66 \mathrm{e}-09$ & $9.41 \mathrm{e}-09$ \\
$1.00 \mathrm{e}-09$ & $4.04 \mathrm{e}-08$ & $1.00 \mathrm{e}-07$ \\
$1.00 \mathrm{e}-10$ & $1.52 \mathrm{e}-07$ & $5.67 \mathrm{e}-07$ \\
$1.00 \mathrm{e}-11$ & $1.46 \mathrm{e}-06$ & $5.23 \mathrm{e}-06$ \\
$1.00 \mathrm{e}-12$ & $3.94 \mathrm{e}-05$ & $1.07 \mathrm{e}-04$ \\
$1.00 \mathrm{e}-13$ & $3.93 \mathrm{e}-04$ & $1.12 \mathrm{e}-03$ \\
$1.00 \mathrm{e}-14$ & $1.73 \mathrm{e}-03$ & $5.84 \mathrm{e}-03$ \\
\hline
\end{tabular}

\section{2 İrdeleme: Hammer Projeksiyonu}

Yeryüzünün tamamının gösterimi için kullanılan alan koruyan gerçek anlamda olmayan azimutal bir projeksiyon olan Hammer projeksiyonunda dört kısmi türev de sifirdan farklıdır. Bu nedenle uygun $t$ parametre değeri ve nümerik türevin türeve ne kadar yakınsadığının araştırılması için uygun eşitliklere sahiptir.

$$
\begin{aligned}
& x=\frac{2 R \sqrt{2} \cos \varphi \sin \frac{\lambda}{2}}{\sqrt{1+\cos \varphi \cos \frac{\lambda}{2}}} \\
& y=\frac{2 R \sqrt{2} \sin \varphi}{\sqrt{1+\cos \varphi \cos \frac{\lambda}{2}}}
\end{aligned}
$$

Tablo 3: Tanjant fonksiyonu için nümerik türev analizi

\begin{tabular}{l|l|l}
\hline$t$ & Ortalama fark & Maks. fark \\
\hline $1.00 \mathrm{e}-02$ & $3.07 \mathrm{e}-02$ & $1.49 \mathrm{e}+00$ \\
$1.00 \mathrm{e}-03$ & $3.05 \mathrm{e}-04$ & $1.47 \mathrm{e}-02$ \\
$1.00 \mathrm{e}-04$ & $3.05 \mathrm{e}-06$ & $1.47 \mathrm{e}-04$ \\
$1.00 \mathrm{e}-05$ & $3.05 \mathrm{e}-08$ & $1.47 \mathrm{e}-06$ \\
$1.00 \mathrm{e}-06$ & $2.98 \mathrm{e}-10$ & $4.84 \mathrm{e}-09$ \\
$1.00 \mathrm{e}-07$ & $4.07 \mathrm{e}-09$ & $7.50 \mathrm{e}-08$ \\
$1.00 \mathrm{e}-08$ & $4.30 \mathrm{e}-08$ & $7.42 \mathrm{e}-07$ \\
$1.00 \mathrm{e}-09$ & $5.60 \mathrm{e}-07$ & $1.01 \mathrm{e}-05$ \\
$1.00 \mathrm{e}-10$ & $6.47 \mathrm{e}-07$ & $1.08 \mathrm{e}-05$ \\
$1.00 \mathrm{e}-11$ & $4.60 \mathrm{e}-06$ & $4.37 \mathrm{e}-05$ \\
$1.00 \mathrm{e}-12$ & $5.89 \mathrm{e}-04$ & $1.03 \mathrm{e}-02$ \\
$1.00 \mathrm{e}-13$ & $5.39 \mathrm{e}-03$ & $9.63 \mathrm{e}-02$ \\
$1.00 \mathrm{e}-14$ & $6.36 \mathrm{e}-03$ & $1.32 \mathrm{e}-01$ \\
\hline
\end{tabular}

Kısmi türevler $(R=1$ alınarak):

$$
\begin{aligned}
& \frac{\partial x}{\partial \varphi}=-\sqrt{2}\left(\frac{\sin \varphi \sin \frac{\lambda}{2}\left(2+\cos \varphi \cos \frac{\lambda}{2}\right)}{\left(1+\cos \varphi \cos \frac{\lambda}{2}\right)^{3 / 2}}\right) \\
& \frac{\partial y}{\partial \varphi}=\frac{\cos \varphi\left(2+\cos \varphi \cos \frac{\lambda}{2}\right)+\cos \frac{\lambda}{2}}{\sqrt{2}\left(1+\cos \varphi \cos \frac{\lambda}{2}\right)^{3 / 2}} \\
& \frac{\partial x}{\partial \lambda}=\frac{\cos \varphi \cos \frac{\lambda}{2}\left(2+\cos \varphi \cos \frac{\lambda}{2}\right)+\cos ^{2} \varphi}{\sqrt{2}\left(1+\cos \varphi \cos \frac{\lambda}{2}\right)^{3 / 2}} \\
& \frac{\partial y}{\partial \lambda}=\frac{\sin \varphi \cos \varphi \sin \frac{\lambda}{2}}{2 \sqrt{2}\left(1+\cos \varphi \cos \frac{\lambda}{2}\right)^{3 / 2}}
\end{aligned}
$$

Uygun $t$ değeri araştırmak ve nümerik türevin türevden sapmalarını belirlemek için $0 \leq \varphi \leq 85^{\circ} 0 \leq \lambda \leq 180^{\circ}$ aralı̆̆ında $5^{\circ}$ aralıklarla bir veri seti oluşturulmuş, değişen $t$ değerlerinde türev ile nümerik türev farklarının mutlak değer olarak ortalamaları hesaplanmıştır (Tablo 4). Tabloda her 
kısmi türev için tüm verilerdeki farkların işaretsiz ortalamaları ve dört kısmi türev için bulunan ortalama farkların ortalamaları görülmektedir ${ }^{1}$. $\mathrm{Bu}$ irdelemede en uygun $t$ değerinin $10^{-6}$ civarında oluştuğu, nümerik türev değerlerinin ise sekiz basamak doğruluğa ulaştığ1 görülmektedir. Trigonometrik fonksiyonların sıklıkla kullanıldığ projeksiyon eşitlikleri için $t=10^{-6}$ uygun bir değer olarak öne çıkmaktadır.

\section{Projeksiyon Deformasyonları}

Harita projeksiyonlarında bir nokta etrafinda diferansiyel anlamda oluşan doğrusal ve alansal ölçek değişimlerine ya da alan, açı ve uzunluk değişimlerine deformasyon denir. Eğri yüzeyin (küre ya da elipsoit) düzleme dönüşümü sonucunda şekil bozulmaları kaçınılmazdır. Orijinal yüzeyde bir nokta merkez alınarak çizilecek yarıçapı bir birim kabul edilen sonsuz küçük bir dairenin izdüşümü projeksiyon düzleminde elips şeklindedir. Bu elips, deformasyon elipsi ya da Tissot Endikatrisi olarak adlandırılır. Elipsin büyük ve küçük yarı eksenleri ile büyük yarı eksenin yatay koordinat ekseninden yapmış olduğu açı $(a, b, \gamma)$ endikatris elemanlarıdır. Elipsin büyük yarı ekseni (a) maksimum uzunluk deformasyonuna, küçük yarı ekseni de minimum uzunluk deformasyonuna (b) karşılık gelir.

Bir harita projeksiyonunda coğrafi ağın izdüşümü ortogonal ise deformasyon elipsi yarı eksenleri meridyen ve paraleller doğrultusunda olur. Bu durumda maksimum ve minimum uzunluk deformasyon yönleri meridyen ve paraleller ile aynı doğrultudadır. A $\breve{g} ı n$ izdüşümü ortogonal değil ise maksimum ve minimum deformasyon doğrultuları meridyen ve paralellerden farklı doğrultudadır.

Herhangi bir doğrultudaki uzunluk deformasyonu ise izdüşüm yüzeyi ve orijinal yüzeydeki diferansiyel uzunlukların oranından elde edilir. Diferansiyel uzunluk elemanları Gauss'un birinci temel biçimi ile ifade edilir. Küreden düzleme herhangi bir projeksiyon

\footnotetext{
${ }^{1}$ Hesaplamalar yazar tarafından geliştirilen yazılım ile yapılmıştır.
}

için bu oran aşağıdaki gibidir.

$$
\begin{aligned}
m^{2} & =\frac{\overline{d s}^{2}}{d s^{2}}=\frac{d x^{2}+d y^{2}}{R^{2} d \varphi^{2}+R^{2} \cos ^{2} \varphi d \lambda^{2}} \\
& =\frac{e d \varphi^{2}+2 f d \varphi d \lambda+g d \lambda^{2}}{E d \varphi^{2}+2 F d \varphi d \lambda+G d \lambda^{2}}
\end{aligned}
$$

$m$ değeri nokta konumuna ve yöne bağlı olarak değişir. Tissot'a göre bir nokta etrafındaki uzunluk deformasyonunun en büyük ve en küçük olduğu birbirine dik iki doğrultu vardır. Bunlar ana deformasyon yönleri olarak adlandirılır (Bildirici, 2018; Hoschek, 1984).

Coğrafi koordinatlar parametre seçilirse küre yüzeyi için temel büyüklükler aşağıdaki gibidir.

$$
E=R^{2} \quad F=0 \quad G=R^{2} \cos ^{2} \varphi
$$

Projeksiyon düzleminde orijinal yüzey parametrelerine göre düzenlenmiş Gauss temel büyüklükleri:

$$
\begin{aligned}
e & =\left(\frac{\partial x}{\partial \varphi}\right)^{2}+\left(\frac{\partial y}{\partial \varphi}\right)^{2} \\
f & =\left(\frac{\partial x}{\partial \varphi}\right)\left(\frac{\partial x}{\partial \lambda}\right)+\left(\frac{\partial y}{\partial \varphi}\right)\left(\frac{\partial y}{\partial \lambda}\right) \\
g & =\left(\frac{\partial x}{\partial \lambda}\right)^{2}+\left(\frac{\partial y}{\partial \lambda}\right)^{2}
\end{aligned}
$$

Meridyen ve paraleller yönündeki uzunluk deformasyonları:

$$
\begin{aligned}
& h=\sqrt{\frac{e}{E}}=\frac{1}{R} \sqrt{\left(\frac{\partial x}{\partial \varphi}\right)^{2}+\left(\frac{\partial y}{\partial \varphi}\right)^{2}} \\
& k=\sqrt{\frac{g}{G}}=\frac{1}{R \cos \varphi} \sqrt{\left(\frac{\partial x}{\partial \lambda}\right)^{2}+\left(\frac{\partial y}{\partial \lambda}\right)^{2}}
\end{aligned}
$$

Normal konumlu projeksiyonlarda meridyen ve paralellerin izdüşümü ortogonal ise $f=0$ olur. Bu durumda ana deformasyon yönleri meridyenler ve paraleller ile çakışı olur. $h$ ve $k$ (10) eşitliğinin en büyük ve en küçük değerlerine eşittir. Bu, özel bir durum olup, genel eşitlikleri elde etmek için (10) eşitliği yöne bağlı düzenlenip türevi sıfıra eşitlenir. Buradan ana deformasyon büyüklükleri $(a, b)$ elde edilir.

$$
\begin{aligned}
K^{2} & =\frac{1}{a^{2}} \frac{1}{b^{2}}=\frac{E G-F^{2}}{e g-f^{2}} \\
2 H^{2} & =\frac{1}{a^{2}}+\frac{1}{b^{2}}=\frac{E g-2 F f+G e}{e g-f^{2}}
\end{aligned}
$$


Tablo 4: Hammer projeksiyonunda nümerik türev türev farkları

\begin{tabular}{l|l|l|l|l|l}
\hline$t$ & $\partial x / \partial \varphi$ & $\partial x / \partial \lambda$ & $\partial y / \partial \varphi$ & $\partial y / \partial \lambda$ & Genel ort. \\
\hline $1.00 \mathrm{E}-03$ & $6.58 \mathrm{E}-08$ & $2.24 \mathrm{E}-08$ & $1.09 \mathrm{E}-07$ & $2.48 \mathrm{E}-09$ & $4.99 \mathrm{E}-08$ \\
$1.00 \mathrm{E}-04$ & $1.50 \mathrm{E}-08$ & $1.03 \mathrm{E}-08$ & $1.67 \mathrm{E}-08$ & $8.58 \mathrm{E}-10$ & $1.07 \mathrm{E}-08$ \\
$1.00 \mathrm{E}-05$ & $1.54 \mathrm{E}-08$ & $1.02 \mathrm{E}-08$ & $1.57 \mathrm{E}-08$ & $8.43 \mathrm{E}-10$ & $\mathbf{1 . 0 5 E - 0 8}$ \\
$1.00 \mathrm{E}-06$ & $1.54 \mathrm{E}-08$ & $1.02 \mathrm{E}-08$ & $1.57 \mathrm{E}-08$ & $8.50 \mathrm{E}-10$ & $\mathbf{1 . 0 5 E - 0 8}$ \\
$1.00 \mathrm{E}-07$ & $1.54 \mathrm{E}-08$ & $1.00 \mathrm{E}-08$ & $1.58 \mathrm{E}-08$ & $9.76 \mathrm{E}-10$ & $\mathbf{1 . 0 5 E - 0 8}$ \\
$1.00 \mathrm{E}-08$ & $2.05 \mathrm{E}-08$ & $1.30 \mathrm{E}-08$ & $1.54 \mathrm{E}-08$ & $3.55 \mathrm{E}-09$ & $1.31 \mathrm{E}-08$ \\
$1.00 \mathrm{E}-09$ & $9.70 \mathrm{E}-08$ & $6.63 \mathrm{E}-08$ & $5.18 \mathrm{E}-08$ & $3.41 \mathrm{E}-08$ & $6.23 \mathrm{E}-08$ \\
$1.00 \mathrm{E}-10$ & $8.22 \mathrm{E}-07$ & $7.43 \mathrm{E}-07$ & $4.99 \mathrm{E}-07$ & $3.43 \mathrm{E}-07$ & $6.02 \mathrm{E}-07$ \\
$1.00 \mathrm{E}-11$ & $1.04 \mathrm{E}-05$ & $7.99 \mathrm{E}-06$ & $5.82 \mathrm{E}-06$ & $3.63 \mathrm{E}-06$ & $6.97 \mathrm{E}-06$ \\
$1.00 \mathrm{E}-12$ & $8.59 \mathrm{E}-05$ & $6.88 \mathrm{E}-05$ & $5.28 \mathrm{E}-05$ & $3.43 \mathrm{E}-05$ & $6.04 \mathrm{E}-05$ \\
$1.00 \mathrm{E}-13$ & $8.63 \mathrm{E}-04$ & $7.20 \mathrm{E}-04$ & $4.43 \mathrm{E}-04$ & $3.36 \mathrm{E}-04$ & $5.91 \mathrm{E}-04$ \\
$1.00 \mathrm{E}-14$ & $9.38 \mathrm{E}-03$ & $6.82 \mathrm{E}-03$ & $5.47 \mathrm{E}-03$ & $3.18 \mathrm{E}-03$ & $6.22 \mathrm{E}-03$ \\
\hline
\end{tabular}

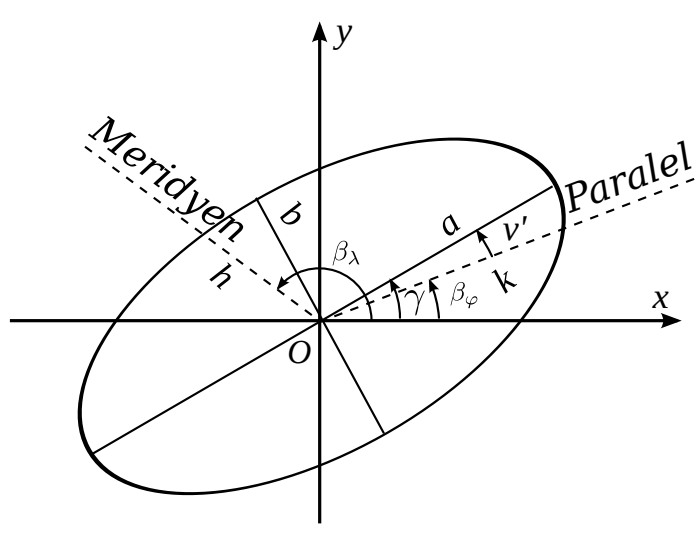

Şekil 2: Endikatris elemanları

$$
\begin{aligned}
& \frac{1}{a}=H^{2}-\sqrt{H^{4}-K^{2}} \\
& \frac{1}{b}=H^{2}+\sqrt{H^{4}-K^{2}}
\end{aligned}
$$

Burada $K$ deformasyon ölçütü, $H$ ise ortalama deformasyon olarak adlandırılır. Alan koruyan projeksiyonlarda $K=1$, konform projeksiyonlarda $K=H^{2}$ olur (Hoschek, 1984).

Ana deformasyon doğrultuları meridyen ve paraleller yönünde değil ise endikatrisin büyük ekseninin $x$ ekseninden yaptığı açı için önce paralel izdüşümünün açısı yardımıyla endikatris yönü belirlenir (Şekil 2).

$$
\begin{aligned}
\tan \beta_{\varphi} & =\frac{\frac{\partial y}{\partial \lambda}}{\frac{\partial x}{\partial \lambda}} \\
\sin v^{\prime} & = \pm \sqrt{\frac{1-a^{2} / k^{2}}{1-a^{2} / b^{2}}} \\
\gamma & =\beta_{\varphi}+\operatorname{signum}(f) v^{\prime}
\end{aligned}
$$

$v^{\prime}$ açısının işareti projeksiyon düzlemindeki temel büyüklük $f$ 'in işareti ile aynı alınmalıdır (Boùùaert vd., 2016; Bildirici, 2016).

Endikatris elemanları Laskowski yöntemi (Laskowski, 1989) ile de bulunabilir. Burada (1) denklemleri ile tanımlanan dönüşümün doğrusallaştırılmış halini ifade eden A matrisinin Single Value Decomposition (SVD) yöntemi ile çarpanlara ayrılmasından yararlanılır.

$$
\begin{aligned}
& \mathbf{A}=\left[\begin{array}{lll}
\frac{1}{\sqrt{G}} \frac{\partial x}{\partial \lambda} & \frac{1}{\sqrt{E}} \frac{\partial x}{\partial \varphi} \\
\frac{1}{\sqrt{G}} \frac{\partial y}{\partial \lambda} & \frac{1}{\sqrt{E}} \frac{\partial y}{\partial \varphi}
\end{array}\right]=\mathbf{U D V}^{T} \\
& \mathbf{D}=\left[\begin{array}{cc}
d_{1} & 0 \\
0 & d_{2}
\end{array}\right] \quad \mathbf{U}=\left[\begin{array}{ll}
u_{11} & u_{12} \\
u_{21} & u_{22}
\end{array}\right]
\end{aligned}
$$

Endikatris elemanları:

$$
\begin{gathered}
a=d_{1} \\
b=d_{2} \\
\tan \gamma=\frac{u_{21}}{u_{11}}
\end{gathered}
$$

Burada V matrisinin bir rolü bulunmamaktadır. Alan deformasyonu ve maksimum yön deformasyonu:

$$
p=a b
$$




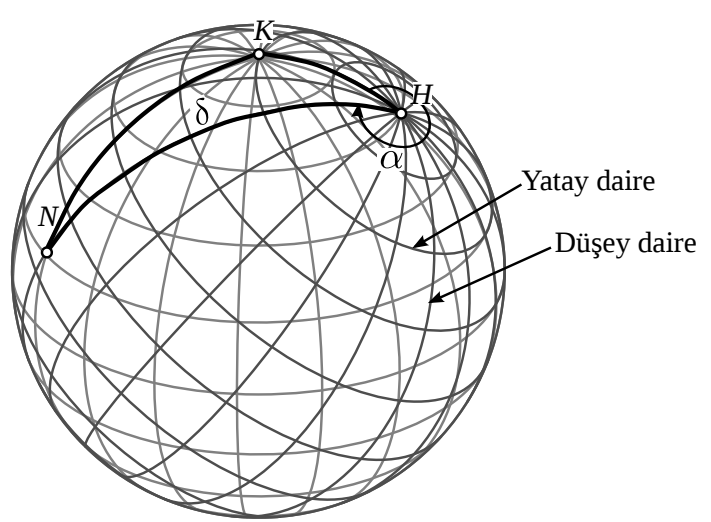

Şekil 3: Projeksiyon yüzeyinin eğik konumlu olması

Açı deformasyonu:

$$
\sin \omega=\frac{a-b}{a+b} \quad w=2 \omega
$$

Coğrafi ağın ortogonal olmaması halinde meridyen ve paralellerin arasında oluşan açı ise aşağıdaki eşitlikle bulunur.

$$
\sin \Omega=\frac{a b}{h k}
$$

Verilen bir nokta ya da noktalar için endikatris elemanlarmın belirlenmesi gerektiğinde de kısmi türevler (7) eşitliklerinden yararlanılarak nümerik olarak da belirlenebilir.

\section{$5 \quad$ Projeksiyon Yüzeyinin Eğik Olması}

Projeksiyon yüzeyinin normal konumlu olmaması halinde coğrafi koordinat sisteminin asal noktaya göre $(H)$ döndürülmesi gerekir. Burada asal nokta, normal konumlu projeksiyonlarda kuzey kutup noktası yerine geçen, azimutal projeksiyonlarda düzlemin referans yüzeyine teğet olduğu nokta, silindirik ve konik projeksiyonlarda ise koni/silindir ekseninin projeksiyon yüzeyini deldiği noktadır. Burada coğrafi koordinatlardan, küresel kutupsal koordinatlara geçiş ( $\alpha$, $\delta)$ kürede ikinci temel ödev çözümü ile gerçekleştirilir (Şekil 3). İkinci temel ödev çözümü $K H N$ küresel üçgeninin çözümünden başka bir şey değildir. $\Delta \lambda=\lambda_{N}-\lambda_{H}$ alınır.

$\cos \delta=\sin \varphi_{H} \sin \varphi_{N}+\cos \varphi_{H} \cos \varphi_{N} \cos \Delta \lambda$

$$
\tan \alpha=\frac{\sin \left(\lambda_{N}-\lambda_{H}\right)}{\cos \varphi_{H} \tan \varphi_{N}-\sin \varphi_{H} \cos \Delta \lambda}
$$

Burada elde edilen $\alpha$, boylam yerine, $\delta$ ise kutup uzaklı̆̆ $\left(90^{\circ}-\varphi\right)$ yerine geçmektedir. Bu büyüklükler normal konumlu eşitliklerde enlem/kutup uzaklı̆̆ ve boylam yerine alınarak eğik konumlu projeksiyon uygulamaları gerçekleştirilir.

Asal noktaya göre oluşan koordinat sisteminde meridyenlere karşılık gelen eğriler düşey daire, paralellere karşllı gelen eğrilere de yatay daireler adı verilir.

Eğik konumlu projeksiyonlarda deformasyonların incelenmesi için kısmi türevlerin parametre dönüşümü ile belirlenmesi gerekir. $\mathrm{Bu}$ durumda harita projeksiyonunu tanımlayan fonksiyon çiftleri aşağıdaki gibidir.

$$
\begin{aligned}
& x=x(\alpha(\varphi, \lambda), \delta(\varphi, \lambda)) \\
& y=y(\alpha(\varphi, \lambda), \delta(\varphi, \lambda))
\end{aligned}
$$

Kısmi türevler aşağıdaki gibi elde edilir ve deformasyon değerlerinin elde edilmesi için kullanılır.

$$
\begin{aligned}
& \frac{\partial x}{\partial \varphi}=\frac{\partial x}{\partial \alpha} \frac{\partial \alpha}{\partial \varphi}+\frac{\partial x}{\partial \delta} \frac{\partial \delta}{\partial \varphi} \\
& \frac{\partial x}{\partial \lambda}=\frac{\partial x}{\partial \alpha} \frac{\partial \alpha}{\partial \lambda}+\frac{\partial x}{\partial \delta} \frac{\partial \delta}{\partial \lambda} \\
& \frac{\partial y}{\partial \varphi}=\frac{\partial y}{\partial \alpha} \frac{\partial \alpha}{\partial \varphi}+\frac{\partial y}{\partial \delta} \frac{\partial \delta}{\partial \varphi} \\
& \frac{\partial y}{\partial \lambda}=\frac{\partial y}{\partial \alpha} \frac{\partial \alpha}{\partial \lambda}+\frac{\partial y}{\partial \delta} \frac{\partial \delta}{\partial \lambda}
\end{aligned}
$$

Örnek: Eğik konumlu uzunluk koruyan azimutal projeksiyon aşağıdaki gibi tanımlıdır.

$$
\begin{aligned}
& x=R \delta \sin \alpha \\
& y=R \delta \cos \alpha
\end{aligned}
$$

$\delta=\arccos \left(\sin \varphi_{H} \sin \varphi+\cos \varphi_{H} \cos \varphi \cos \Delta \lambda\right)$

$$
\begin{gathered}
\alpha=\arctan \left(\frac{\sin \Delta \lambda}{\tan \varphi \cos \varphi_{H}-\sin \varphi_{H} \cos \Delta \lambda}\right) \\
\Delta \lambda=\lambda-\lambda_{H}
\end{gathered}
$$

$\mathrm{Bu}$ eşitliklerde asal nokta keyfi olarak, genellikle projeksiyonun uygulanacağı bölgenin ortasında seçilir. Burada $x$ ekseni asal noktadan geçen meridyenin izdüşümüne çakışık olarak alınmıştır. Kısmi türevler: 


$$
\begin{aligned}
\frac{\partial \delta}{\partial \varphi} & =-\frac{\cos \varphi \sin \varphi_{H}-\sin \varphi \cos \varphi_{H} \cos \Delta \lambda}{A} \\
\frac{\partial \delta}{\partial \lambda} & =\frac{\cos \varphi \cos \varphi_{H} \sin \Delta \lambda}{A} \\
\frac{\partial \alpha}{\partial \varphi} & =-\frac{\sin \Delta \lambda \cos \varphi_{H}}{\cos ^{2} \varphi\left(\sin ^{2} \Delta \lambda+B^{2}\right)} \\
\frac{\partial \alpha}{\partial \lambda} & =\frac{B \cos \left(\lambda-\lambda_{H}\right)-\sin ^{2} \Delta \lambda \sin \varphi_{H}}{\sin ^{2} \Delta \lambda+B^{2}}
\end{aligned}
$$

$A=\sqrt{1-\left(\sin \varphi_{H} \sin \varphi+\cos \varphi_{H} \cos \varphi \cos \Delta \lambda\right)^{2}}$

$B=\tan \varphi \cos \varphi_{H}-\sin \varphi_{H} \cos \Delta \lambda$

$$
\begin{array}{lll}
\frac{\partial x}{\partial \delta}=R \sin \alpha, & \frac{\partial x}{\partial \alpha}=R \delta \cos \alpha \\
\frac{\partial y}{\partial \delta}=R \cos \alpha, & \frac{\partial y}{\partial \alpha}=-R \delta \sin \alpha
\end{array}
$$

\section{Ara Değişken Kullanılan Projeksiyonlar}

Gerçek anlamda olmayan silindirik projeksiyonların bazılarında enleme bağlı olarak genellikle kapalı bir fonksiyonla tanımlanan bir ara değişken kullanılır. Kapalı fonksiyonlar söz konusu ise ara değişkenin enleme bağlı olarak bulunması iteratif çözüm gerektirir. Örnek olarak Mollweide projeksiyonunu ${ }^{2}$ ele alalım (Şekil 4).

$$
\begin{gathered}
x=\frac{2 \sqrt{2}}{\pi} R\left(\lambda-\lambda_{0}\right) \cos \theta \\
y=\sqrt{2} R \sin \theta \\
2 \theta+\sin 2 \theta=\pi \sin \varphi
\end{gathered}
$$

Burada $\lambda_{0}, \quad x$ ekseninin çakışık alınacă̆ meridyen (orta meridyen) olup dünya haritalarında genel olarak 0 alınır. $\theta$ açısı ise Newton-Raphson iterasyonu ile bulunabilir (Bildirici, 2018; Snyder, 1987). Kapalı eşitlik $f(\theta)=0$ şeklinde düzenlenirse:

$$
\begin{gathered}
f(\theta)=2 \theta+\sin 2 \theta-\pi \sin \varphi=0 \\
f^{\prime}(\theta)=2+2 \cos 2 \theta
\end{gathered}
$$

\footnotetext{
${ }^{2}$ Alman K.B. Mollweide (1774-1825) tarafindan geliştirilmiş yeryüzünün tamamının gösterimi için kullanılan alan koruyan gerçek anlamda olmayan silindirik projeksiyon
}

iterasyon,

$$
\theta_{i+1}=\theta_{i}-\frac{f\left(\theta_{i}\right)}{f^{\prime}\left(\theta_{i}\right)}
$$

eşitliğinde ikinci terim yeterince küçük oluncaya kadar devam ettirilir. Başlangıç değeri aşă̆ıdaki gibi seçilebilir.

$$
\theta_{0}=\frac{\pi \sin \varphi-\sin 2 \varphi}{2}
$$

Çözüm Halley yöntemi ile de yapılabilir (Weissstein, 2019).

$$
\theta_{i+1}=\theta_{i}-\frac{2 f\left(\theta_{i}\right) f^{\prime}\left(\theta_{i}\right)}{2\left(f^{\prime}\left(\theta_{i}\right)\right)^{2}-f\left(\theta_{i}\right) f^{\prime \prime}\left(\theta_{i}\right)}
$$

Ya da

$$
\theta_{i+1}=\theta_{i}-\frac{f\left(\theta_{i}\right)}{f^{\prime}\left(\theta_{i}\right)-\frac{f\left(\theta_{i}\right) f^{\prime \prime}\left(\theta_{i}\right)}{2 f^{\prime}\left(\theta_{i}\right)}}
$$

$\mathrm{Bu}$ tür projeksiyonlarda enleme bağlı kısmi türevler aşağıdaki gibidir.

$$
\begin{aligned}
& \frac{\partial x}{\partial \varphi}=\frac{\partial x}{\partial \theta} \frac{\partial \theta}{\partial \varphi} \\
& \frac{\partial y}{\partial \varphi}=\frac{\partial y}{\partial \theta} \frac{\partial \theta}{\partial \varphi}
\end{aligned}
$$

Kapalı fonksiyonun türevi $f(x, y)=0$ olmak üzere,

$$
\frac{d y}{d x}=f^{\prime}(x, y)=-\frac{f_{x}^{\prime}(x, y)}{f_{y}^{\prime}(x, y)}
$$

Burada:

$f_{x}^{\prime}(x, y): y$ sabit kabul edilip $x$ e göre alınan türev

$f_{y}^{\prime}(x, y): x$ sabit kabul edilip $y$ e göre alınan türev

Mollweide projeksiyonu için uygulanırsa:

$$
\begin{gathered}
f(\varphi, \theta)=2 \theta+\sin 2 \theta-\pi \sin \varphi=0 \\
\frac{d \theta}{d \varphi}=-\frac{f_{\varphi}^{\prime}(\varphi, \theta)}{f_{\theta}^{\prime}(\varphi, \theta)}=\frac{\pi \cos \varphi}{2+2 \cos 2 \theta}
\end{gathered}
$$

Kısmi türevler:

$$
\begin{aligned}
& \frac{\partial x}{\partial \varphi}=\frac{-2 \sqrt{2}}{\pi}\left(\lambda-\lambda_{0}\right) \sin \theta \frac{\pi \cos \varphi}{2+2 \cos 2 \theta} \\
& \frac{\partial x}{\partial \lambda}=\frac{2 \sqrt{2}}{\pi} \cos \theta \\
& \frac{\partial y}{\partial \varphi}=\sqrt{2} \cos \theta \frac{\pi \cos \varphi}{2+2 \cos 2 \theta} \\
& \frac{\partial y}{\partial \lambda}=0
\end{aligned}
$$




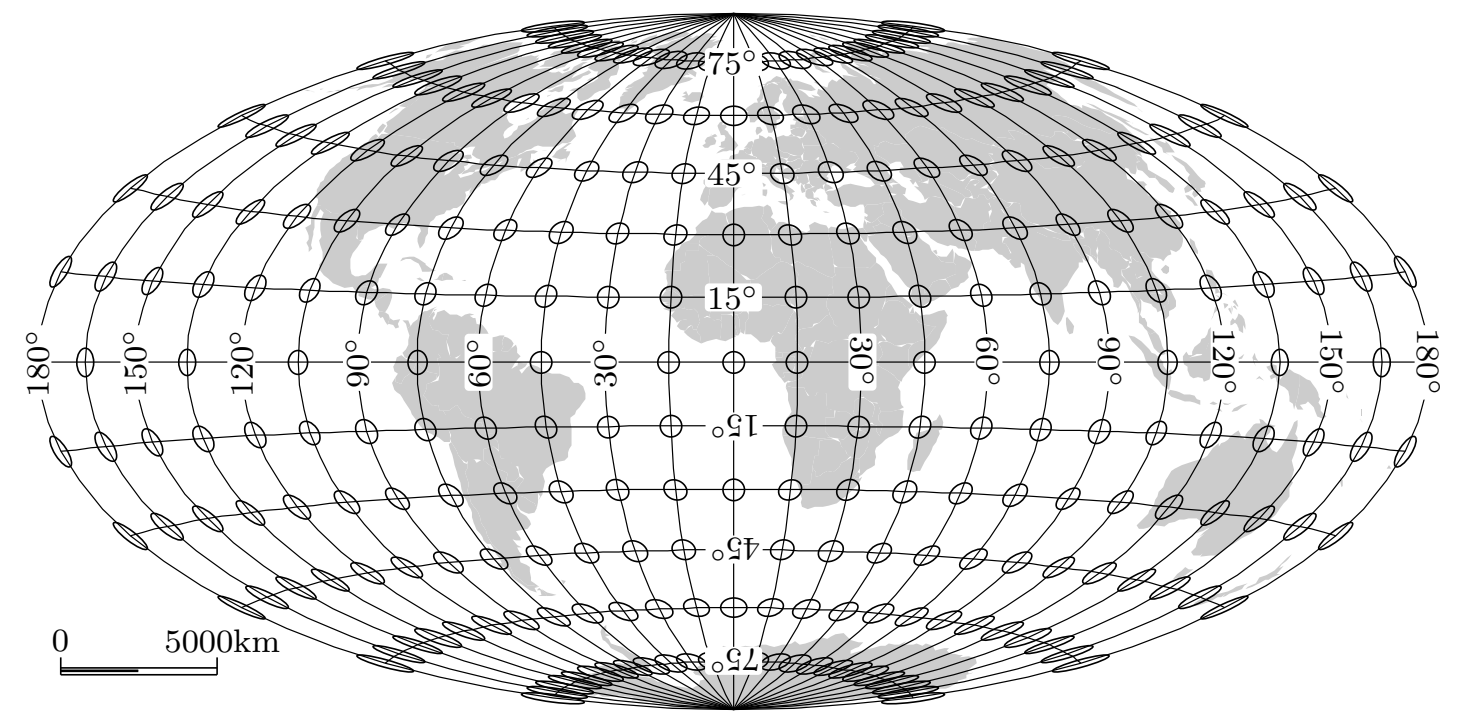

Şekil 4: Mollweide projeksiyonunda endikatris gösterimi ile birlikte dünya

Benzer şekilde çok sayıda gerçek anlamda olmayan silindirik projeksiyon vardır (Daha fazla bilgi için: Uçar vd. (2011), Snyder ve Voxland (1989), Snyder (1993), Bugayevskiy ve Snyder (1995)).

\section{Nümerik Ters Projeksiyon}

Harita projeksiyonlarında (1) eşitlikleri biliniyorken (2) eşitliklerinin elde edilmesi nümerik olarak gerçekleştirilebilir. Winkel Tripel $^{3}$ gibi bazı projeksiyonlarda doğrusal olmayan denklemlerin çözümü gerektiğinden yalnızca nümerik yaklaşım mümkündür. $\mathrm{Bu}$ amaçla Newton-Raphson yöntemi kullanılabilir (Burden ve Faires, 2001; Yang vd., 1999). Seçilen bir başlangıç değer çiftine bağlı olarak $\left(\varphi_{0}, \lambda_{0}\right)$,

$$
\begin{aligned}
& \mathbf{F}=\left[\begin{array}{l}
x\left(\varphi_{i}, \lambda_{i}\right)-x \\
y\left(\varphi_{i}, \lambda_{i}\right)-y
\end{array}\right] \\
& \mathbf{J}=\left[\begin{array}{ll}
\frac{\partial x}{\partial \varphi} & \frac{\partial x}{\partial \lambda} \\
\frac{\partial y}{\partial \varphi} & \frac{\partial y}{\partial \lambda}
\end{array}\right] \\
& \mathbf{X}=\left[\begin{array}{l}
\varphi \\
\lambda
\end{array}\right]
\end{aligned}
$$

olmak üzere iterasyon

$$
\mathbf{X}_{i+1}=\mathbf{X}_{i}-\mathbf{J}_{i}^{-1} \mathbf{F}_{i}
$$

\footnotetext{
${ }^{3}$ Oswald Winkel (1873-1953) tarafindan 1921'de sunulmuş yeryüzünün tamamını gösterimine yönelik gerçek anlamda olmayan bir projeksiyon
}

X matrisinde değişim yeterince küçük oluncaya kadar devam ettirilir. Bilgisayar ortaminda double precision reel sayı kullanılması halinde maksimum basamak doğruluğuna ulaşmak için iterasyon,

$$
\mathbf{J}_{i}^{-1} \mathbf{F}_{i} \leq\left[\begin{array}{c}
10^{-14} \\
10^{-14}
\end{array}\right]
$$

oluncaya kadar devam ettirilmelidir.

Ters dönüşümde dönüşümü yapılan noktanın orta meridyen ya da Ekvator üzerinde olması gibi özel durumların dikkate alınması gerekir. Örneğin Mollweide projeksiyonu gibi (Şekil 4) gerçek anlamlı olmayan silindirik projeksiyonlarda; projeksiyon düzleminde düzlem Kartezyen koordinat ve coğrafi koordinat sistemlerinin orijini çakışık ise, düzlem koordinatlardan birinin sıfır olması durumunda istenen coğrafi koordinatlardan biri de sıfır olacaktır. Bu durumun iterasyon öncesi kontrol edilmesi gerekir.

Bu tür projeksiyonlarda $x=0, y \neq 0$ ise $\lambda=0$ olur, enlem ise aşağıdaki belirlenir.

$$
\varphi_{i+1}=\varphi_{i}-\frac{y\left(\varphi_{i}, 0\right)-y}{\left(\frac{\partial y}{\partial \varphi}\right)_{i}}
$$

$x \neq 0, y=0$ ise nokta ekvator üzerindedir $(\varphi=$ $0)$, boylam aşağıdaki belirlenir.

$$
\lambda_{i+1}=\lambda_{i}-\frac{x\left(0, \lambda_{i}\right)-x}{\left(\frac{\partial x}{\partial \lambda}\right)_{i}}
$$


İterasyon başlangıç değerlerinin seçimi de önemlidir. İncelenen projeksiyona özgü başlangıç değerleri araştırılabilir. Bir çok projeksiyon için geçerli olabilecek değerler aranırsa, kuzeydoğu çeyrek küre orta noktasindan $\left(\varphi=45^{\circ}, \lambda=90^{\circ}\right)$ yararlanılabilir.

Winkel-Tripel projeksiyonu ve diğer bazı gerçek anlamda olmayan silindirik projeksiyonlar için için nümerik ters projeksiyon uygulamalarını Ipbuker ve Bildirici (2005) ve Ipbuker (2009) ele almıştır.

Nümerik ters projeksiyon nümerik türev ile uygulanabilir. $\mathrm{Bu}$ durumda bilgisayar programlama tekniği açısından düz projeksiyon eşitliklerinin kodlanmış olması yeterlidir. Bu şekilde kısmi türevler kolayca hesaplanarak ters projeksiyon nümerik olarak yapılabilir. Bir çok projeksiyon için kodlama yapılan bir programlama çalışmasında ters projeksiyon eşitliklerinin ayrıca kodlanmasına gerek kalmaz. Bildirici (2017) değişik projeksiyonlarda bu yaklaşımın uygulanabilirliğini göstermiştir.

\section{Sonuç}

$\mathrm{Bu}$ çalışmada harita projeksiyonları ile ilgili nümerik analiz konuları uygulamalar eşliğinde ele alınmıştır. Nümerik türevin hangi koşullarda uygulanabilir olduğu irdelenmiş harita projeksiyonları için kullanılabilecek parametre önerileri yapılmıştır. Projeksiyon yüzeyinin eğik olması ve bir yardımcı değişken ile tanımlanmıs projeksiyonlarda deformasyon analizi konusu tartışılmıştır. Son olarak ters projeksiyonun (düzlem koordinatlardan coğrafi koordinatların bulunması) nümerik olarak uygulanması ele alınmıştır.

\section{Kaynaklar}

Bildirici, İ. Ö. (2016). Ortogonal olmayan coğrafi ağlı harita projeksiyonlarında Tissot endikatris elemanlarmn belirlenmesi. Harita Dergisi, (156):13-22.

Bildirici, ̇. Ö. (2017). An iterative approach for inverse transformation of map projections. Cartography and Geographic Information Science, 44(5):463-471.

Bildirici, İ. Ö. (2018). Kartografya: Harita Tasarımı ve Kullanımı için Gerekli Bilim, Sanat ve Teknik. Atlas Akademi Yayınevi, Konya.

Boùùaert, M. C., Baets, B. D., Vervust, S., Neutens, T., Maeyer, P. D., ve de Weghe, N. V. (2016). Computation and visualisation of the accuracy of old maps using differential distortion analysis. International Journal of Geographical Information Science, 30(7):1255-1280.

Bugayevskiy, L. M. ve Snyder, J. (1995). Map projections: A reference manual. CRC Press.

Burden, R. L. ve Faires, J. D. (2001). Numerical analysis.

Hoschek, J. (1984). Matematische Grundlagen der Kartographie. Bibliographisches Institut, Mannheim.

Ipbuker, C. (2009). Inverse transformation for several pseudo-cylindrical map projections using jacobian matrix. International Conference on Computational Science and Its Applications. Springer, 553-564.

Ipbuker, C. ve Bildirici, İ. Ö. (2005). Computer program for the inverse transformation of the winkel projection. Journal of Surveying Engineering, 131(4):125-129.

Laskowski, P. H. (1989). The traditional and modern look at tissot's indicatrix. The American Cartographer, 16(2):123-133.

Snyder, J. P. (1987). Map projections-A working manual, volume 1395 . US Government Printing Office.

Snyder, J. P. (1993). Flattening the Earth. University of Chicago Press, Chicago.

Snyder, J. P. ve Voxland, P. (1989). An Album of Map Projections. US Geological Survey, Washington DC.

Uçar, D., İpbüker, C., ve Bildirici, İ. Ö. (2011). Matematiksel Kartografya. Atlas Yayın Dağıtım, Ankara. 
Weissstein, E. W. (2019). Halley's method. http://mathworld. wolfram.com/ HalleysMethod.html. Giriş: 31.01.2019.

Yang, Q., Snyder, J., ve Tobler, W. (1999). Map projection transformation: principles and applications. CRC Press. 